\title{
MODELING THE NUCLEATION KINETICS AND AEROSOL DYNAMICS OF PARTICLE FORMATION DURING CVD OF SILICON FROM SILANE
}

\author{
M. T. SWIHART, S. NIJHAWAN, M. R. MAHAJAN, S.-M. SUH, and S. L. GIRSHICK \\ University of Minnesota, Dept. of Mechanical Engineering, Minneapolis, MN 55455 USA
}

\section{KEYWORDS}

\author{
Nucleation, Silicon CVD, Silane, Chemical Kinetics
}

\begin{abstract}
Product contamination by gas-phase nucleation within the processing environment often limits the deposition rate that can be obtained during chemical vapor deposition (CVD) of materials for microelectronics applications. A fundamental understanding of how these particles nucleate and grow may allow us to enlarge the process envelope, providing higher growth rates without particle contamination. Since these particles are formed by a series of chemical reactions involving multiple chemical species with formation and breakage of strong chemical bonds, classical homogeneous nucleation theory fails to adequately describe their generation. The work presented here is part of an ongoing effort to develop a generalized framework for describing these chemical nucleation processes. This study focuses on particle formation during thermal CVD of silicon from silane. We have modeled particle nucleation, growth, and transport in both low-pressure and atmospheric-pressure CVD of silicon from silane, and present results of those modeling efforts here.
\end{abstract}

The nucleation of particles has been modeled by a series of chemical reactions that lead from silane to larger silicon hydrides and finally to hydrogenated silicon clusters that can be considered particles. The thermochemistry and kinetics of these reactions are estimated based on the known reactivity and thermochemistry of the smaller silicon hydrides. A group additivity scheme for estimating the thermodynamic properties of arbitrary silicon hydride clusters was developed by fitting group parameters to the results of the extensive ab initio calculations of Katzer et al (1997). Pressure-dependent rate parameters for reactions of silicon hydrides with up to three silicon atoms were taken from the work of Ho, Coltrin and Breiland (1994). For the larger silicon hydrides, four classes of reversible chemical reactions were considered, as shown in Table 1. The polysilanes produced by these reactions can grow either by eliminating hydrogen to form a silylene (reaction type 1) that then reacts with silane (reverse of reaction type 2), or by reacting directly with silylene (reverse of reaction type 2) produced by thermal decomposition of silane. Silylenes can reversibly isomerize to silenes (reaction type 3), which are thermodynamically more stable than silylenes and are effectively unreactive. Silylenes with the proper geometry can undergo intramolecular insertion to form a ring (reaction type 4). The growth process described above is in competition with polysilane decomposition (reaction type 2). Ring formation plays a key role in the growth process, because once a silicon atom is incorporated into a ring it cannot directly be eliminated by a reaction of type 2 . Ring formation also accounts for the dehydrogenation of the particles, since cyclic compounds have lower silicon-to-hydrogen ratios than acyclic compounds. To our knowledge, this cyclization has not been included in any previous models of silicon hydride chemistry. 
Table 1: Reaction Types

\begin{tabular}{|c|c|c|}
\hline Reaction Type & General Form & Typical Reactions \\
\hline (1) Hydrogen Elimination & silane $\leftrightarrow$ silylene $+\mathrm{H}_{2}$ & $\mathrm{SiH}_{4} \leftrightarrow \mathrm{SiH}_{2}+\mathrm{H}_{2}$ \\
\hline (2) Silylene Elimination & silane $\leftrightarrow$ silane + silylene & $\begin{aligned} & \mathrm{Si}_{2} \mathrm{H}_{6} \leftrightarrow \mathrm{H}_{3} \mathrm{SiSiH}+\mathrm{H}_{2} \\
& \mathrm{Si}_{2} \mathrm{H}_{6} \leftrightarrow \leftrightarrow \mathrm{SiH}_{4}+\mathrm{SiH}_{2} \\
& \mathrm{Si}_{3} \mathrm{H}_{8} \leftrightarrow \mathrm{SiH}_{4}+\mathrm{H}_{3} \mathrm{SiSiH}\end{aligned}$ \\
\hline $\begin{array}{l}\text { (3) Silylene/Silene Isomerization } \\
\text { (4) Ring Formation }\end{array}$ & $\begin{array}{l}\text { silylene } \leftrightarrow \text { silene } \\
\text { acyclic silylene } \leftrightarrow \\
\text { cyclic silane }\end{array}$ & $\begin{aligned} \mathrm{H}_{3} \mathrm{SiSiH} & \leftrightarrow \mathrm{H}_{2} \mathrm{SiSiH}_{2} \\
\mathrm{SiH}_{3} \mathrm{SiH}_{2} \mathrm{SiH} & \leftrightarrow \text { cyclotrisilane } \\
\mathrm{SiH}_{3} \mathrm{SiH}_{2} \mathrm{SiH}_{2} \mathrm{SiH} & \leftrightarrow \text { cyclotetrasilane }\end{aligned}$ \\
\hline
\end{tabular}

Rate parameters for all of these reactions were estimated based on the analogous reactions of silane, disilane, trisilane, and tetrasilane that have been studied both experimentally and theoretically. Reaction mechanisms containing the most thermodynamically stable isomers of the various silicon hydride clusters were constructed. A suitably large silicon hydride (on the order of 10 silicon atoms) was termed a critical nucleus, and the nucleation rate was obtained as the rate of production of this species.

We modelled particle growth and transport in these systems with a moment-type model. A lognormal particle size distribution was assumed, and equations for the first three moments of the volume size distribution were solved. Standard forms were assumed for the convection, diffusion, thermophoresis, and coagulation terms, including the assumption that particles are spherical and always coagulate upon collision. Particle growth rates were calculated from the same surface reaction mechanism used for CVD on flat surfaces, based on the mechanism of Ho, Coltrin and Breiland (1994). The moment model was fully coupled to the momentum, enthalpy, and chemical species equations, including a proper accounting of reactant species depletion due to particle formation.

Low-pressure CVD was simulated in a stagnation-point geometry based on the GEC reference cell configuration. Atmospheric-pressure CVD was simulated in an axisymmetric hot tube. These geometries and conditions correspond to ongoing experiments in our laboratory. A comparison of the model predictions with experimental results for the low-pressure configuration is promising. The model correctly predicts the rapid onset of particle nucleation at critical temperatures and pressures comparable to those observed experimentally.

\section{ACKNOWLEDGEMENTS}

This work was partially supported by the Semiconductor Research Corporation, Advanced Silicon Materials, Inc., and CFD Research Corporation.

\section{REFERENCES}

Ho, P., M. E. Coltrin and W. G. Breiland (1994), "Laser-Induced Fluorescence Measurements and Kinetic Analysis of Si Atom Formation in a Rotating Disk Chemical Vapor Deposition Reactor,"J. Phys.Chem. 98, 10138-10147.

Katzer, G., M. C. Ernst, A. F. Sax and J. Kalcher (1997), "Computational Thermochemistry of Medium-Sized Silicon Hydrides," J. Phys. Chem. A 